\title{
Effect of Preparation Additives on the Antimicrobial Activity and Cytotoxicity of Polypyrrole
}

\author{
Yslaine A. de Almeida, ${ }^{\circledR a}$ Diego F. Bispo, ${ }^{\circledR a}$ Monalisa M. Montalvão, ${ }^{\odot b}$ \\ Karina O. Mota, ${ }^{c}$ Cristiane B. Corrêa ${ }^{\circledR b}$ and Iara F. Gimenez ${ }^{\circledR *} * a$
}

\author{
${ }^{a}$ Departamento de Química, Universidade Federal de Sergipe, \\ Avenida Marechal Rondon, s/n, Jardim Rosa Elze, 49100-000 São Cristóvão-SE, Brazil \\ ${ }^{b}$ Departamento de Morfologia, Universidade Federal de Sergipe, \\ Avenida Marechal Rondon, s/n, Jardim Rosa Elze, 49100-000 São Cristóvão-SE, Brazil \\ ${ }^{c}$ Departamento de Fisiologia, Universidade Federal de Sergipe, \\ Avenida Marechal Rondon, s/n, Jardim Rosa Elze, 49100-000 São Cristóvão-SE, Brazil
}

\begin{abstract}
The search for new biomaterials has increased continuously in recent years and, in this context, the application of polypyrrole (PPy) as a promising material for this function has been reported as a result of positive responses, such as bioactivity and biocompatibility. In this study, PPy was obtained through modifications/adaptations made in methodologies of previous papers, which focused mainly on conductive properties. The use of additives in the synthesis significantly improves the application in the biomedical area, which depends directly on a diversity of structural and morphological parameters. Here, physicochemical characterization was helpful to determine PPy structures and morphologies of the resulting samples. The addition of phytic acid (PA) and methyl orange (MO) directly influenced not only the particle morphology and size, but also the antibacterial activity and cytotoxicity. The results of antibacterial activity against Staphylococcus aureus and Escherichia coli as well as the negligible cytotoxicity of PPy obtained here indicate a wide applicability of the materials in wound dressing and tissue engineering.
\end{abstract}

Keywords: polypyrrole, morphology, antimicrobial activity, biocompatibility

\section{Introduction}

Intrinsically conductive polymers (ICP's) have been extensively investigated due to their electrical, optical, and oxidation properties ${ }^{1-3}$ as well as their good chemical stability. ${ }^{4}$ As a representative of this class of polymers, polypyrrole (PPy), is suitable for several applications such as chemical sensors, ${ }^{5}$ optical devices, ${ }^{6}$ electrochemical supercapacitors ${ }^{7}$ and bactericidal agents. ${ }^{8}{ }^{8}$ However, some applications of PPy are limited by its poor solubility and processability. Thus, these disadvantages can be overcome through the preparation of nanoscale PPy particles using additives such as oxidant or reducing agents as well as surfactants, seeking to improve solubility and to avoid particle agglomeration.

The use of additives is common in the synthesis of PPy, including phytic acid (PA) which is widely used due

*e-mail: Iara.gimenez@gmail.com to its very low toxicity. Thus, the resulting methodology is considered a "green synthesis" 10,11 and it has been reported that PA favors the formation of pores ${ }^{12,13}$ across gel networks composed by primary globular particles. PA can also act as a doping agent yielding conductive hydrogels..$^{10,14,15}$

Azo sulfonic dyes are also employed as modifying agents providing both conductivity and morphological control to the synthesis of PPy. ${ }^{15-17}$ Methyl orange (MO) and ethyl orange (EO) are dyes commonly used for this purpose and are well known to favor the nanotubular and globular morphology, respectively. ${ }^{15}$ Additionally, MO may act as a surfactant ${ }^{17}$ depending on factors such as acid/ basic behavior, ${ }^{18}$ concentration and the addition order of reactant. ${ }^{19-22}$

Previous studies ${ }^{17,23}$ using PPy focused mainly on its electrical properties and the influence of morphology on these properties. However, these methodologies can also produce samples suitable for other types of applications, such as biomedical materials. ${ }^{24}$ Studies in the literature 
evidence that PPy is a promising material for this application due to its bacteriostatic properties, ${ }^{25-27}$ wound healing and muscle tissue regeneration. ${ }^{3,28-30}$ In the absence of additives, PPy performance in biomedical studies was found to be poor, which justifies biomedical studies with additive-containing samples. ${ }^{1,31}$

Biocidal application of antimicrobial materials became an alternative to existing biocides such as antibiotics. ${ }^{32}$ Evaluation of PPy performance against certain pathogenic bacteria showed promising results, mainly due to the presence of positive charges able to interact with the bacterial cell wall., 2,26,32-34 Another important evaluation is in vitro cytotoxicity test, which is an initial test required for biomedical materials.

The novelty of the present study consists in the direct comparison of two chemical polymerization methodologies. ${ }^{35}$ The effect of the different methodologies on the biological properties was evaluated through cytotoxicity test on L929 fibroblast cells and bactericidal activity tests on Gram-positive and Gram-negative bacteria, through the zone of inhibition (ZI) and minimum inhibitory concentration (MIC).

\section{Experimental}

Reagents

Pyrrole $\left(\mathrm{C}_{4} \mathrm{H}_{5} \mathrm{~N}\right) 99 \%$ (Acros Organics, New Jersey, USA) was distilled prior to use, methyl orange $\left(\mathrm{C}_{14} \mathrm{H}_{14} \mathrm{~N}_{3} \mathrm{NaO}_{3} \mathrm{~S}\right)$ (Dinâmica, Indaiatuba, Brazil), ammonium persulfate $\left(\left(\mathrm{NH}_{4}\right)_{2} \mathrm{~S}_{2} \mathrm{O}_{8}\right)$ (Sigma-Aldrich, São Paulo, Brazil), isopropyl alcohol $\left(\mathrm{C}_{3} \mathrm{H}_{8} \mathrm{O}\right)$ P.A. (NEON, Suzano, Brazil), phytic acid $\left(\mathrm{C}_{6} \mathrm{H}_{18} \mathrm{O}_{24} \mathrm{P}_{6}\right) 50 \%$ (v/v) in $\mathrm{H}_{2} \mathrm{O}$ (Sigma-Aldrich, São Paulo, Brazil) were used as received.

\section{Synthesis}

Two methods were developed by adapting previous works reported by Pan et al. ${ }^{35}$ and Ying et al. ${ }^{36}$ respectively and samples were identified as PPy I and PPy II.

\section{Preparation of PPy I}

Two solutions (A and B) were previously prepared. For solution A $3.6 \mathrm{mmol}$ of ammonium persulfate (APS) were dissolved in $1500 \mu \mathrm{L}$ of deionized water, while for solution B $252 \mu \mathrm{L}$ of pyrrole (Py) and $552 \mu \mathrm{L}$ of phytic acid (PA) were dissolved in $1500 \mu \mathrm{L}$ of isopropyl alcohol (IP). Both solutions were cooled down separately to approximately $4{ }^{\circ} \mathrm{C}$ in the freezer, then mixed together with mechanical stirring. Finally, the mixture was placed in a previously cooled Petri dish and kept for $2 \mathrm{~h}$ at rest to allow for the reaction to occur. The PPy preparation scheme for this methodology and the chemical structure of the hydrogel are illustrated in Figure 1.

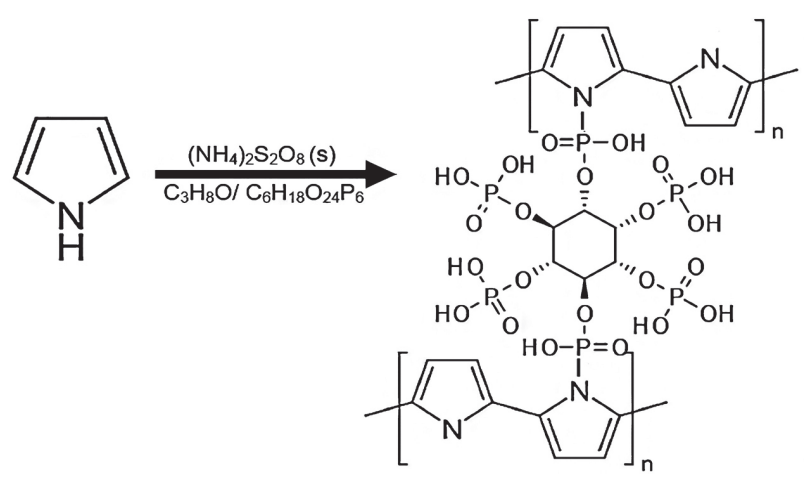

Figure 1. Reaction scheme and chemical structure of the PPy, where phytic acid served as a gelling agent and dopant.

To remove excess ions, acids and by-products, the thin film of PPy gel was further purified by sequential immersion in ethanol $(12 \mathrm{~h})$ and afterwards in deionized water $(24 \mathrm{~h})$. The PPy film was dried at $60^{\circ} \mathrm{C}$ under vacuum in an oven and rehydrated to form a black hydrogel by the addition of $2 \mathrm{~mL}$ of deionized water.

\section{Preparation of PPy II}

A mass corresponding to $5 \mathrm{mmol}$ of ammonium persulfate (APS) was added to $100 \mathrm{~mL}$ of a $5 \mathrm{mmol} \mathrm{L}^{-1}$ methyl orange (MO) aqueous solution followed by magnetic stirring. Then, $5 \mathrm{mmol}$ of pyrrole (Py) and $1 \mathrm{mmol}$ of phytic acid (PA) were added to the solution to proceed with the oxidation while stirring for $5 \mathrm{~min}$. Subsequently, the reaction mixture was stored at $4{ }^{\circ} \mathrm{C}$ for $24 \mathrm{~h}$ to give a slightly viscous black precipitate which was purified with deionized water and freeze-dried.

\section{Characterization methods}

The molecular structure of the PPy was characterized by Fourier transform infrared (FTIR) spectroscopy of $\mathrm{KBr}$ pellets using a Fourier transform infrared spectrotometer (model IR Prestige 21, Shimadzu, Tokyo, Japan). The degree of ordering at medium and long ranges of the samples were characterized using a XRD diffractometer (model LabX XRD-6000, Shimadzu, Tokyo, Japan). The thermal stability of PPy was tested with a thermogravimetry analyzer (model 449 F1 Jupiter, NETZSCH, Selb, Germany) at a heating rate of $10^{\circ} \mathrm{C} \mathrm{min}^{-1}$ from 25 to $800^{\circ} \mathrm{C}$ under nitrogen atmosphere. The mean surface area, volume and pore diameter were determined by nitrogen adsorption, 
using a multipoint Brunauer-Emmet-Teller (BET) and Barrett-Joyner-Halenda (BJH) method using a gas adsorption analyzer (model NOVA 1200e, Quantachrome/ Anton Paar, Graz, Germany).

The morphology of the different PPy samples was analyzed by scanning electron microscopy (SEM) using a microscope (model JSM-5700, Jeol, Tokyo, Japan), with operation between 5 and $15 \mathrm{kV}$, and to complement, transmission electron microscopy (TEM) using a microscope (model JEM 1400Plus, Jeol, Tokyo, Japan), operating at $120 \mathrm{kV}$ voltage. For observation at TEM, the samples were suspended in isopropyl alcohol and deposited on a copper grid coated with a carbon film. TEM images were processed and from the data given, histograms were created for $>150$ particles where we could extract the diameter and calculate the particle size using the public domain software Fiji ImageJ. ${ }^{37}$

\section{Antibacterial activity tests}

The antibacterial activity was determined using the agar diffusion procedure measuring the zone of inhibition (ZI) and minimum inhibitory concentration (MIC). The tests were performed against Gram-positive bacteria (Staphylococcus aureus ATCC 25923) and Gram-negative bacteria (Escherichia coli ATCC 25992) donated by the National Institute for Quality Control in Health (INCQS-Fiocruz), with methodology adapted from Santos et al. ${ }^{38}$

All devices and materials were initially autoclaved at $120^{\circ} \mathrm{C}$ for 15 min to carry out the experiments. For agar diffusions test, in a laminar flow, approximately $30 \mathrm{~mL}$ of the autoclaved nutrient agar solution were deposited onto Petri dishes and kept at rest for $1 \mathrm{~h}$ in order to dry the nutrient medium. Subsequently, with the aid of the autoclaved test tube, perforations were made in the agar, forming a "well" in the center of the plate, and about $200 \mu \mathrm{L}$ of it was added at the bottom to partially cover the well. The bacteria were cultured during $24 \mathrm{~h}$ before inoculation in a brain heart infusion (BHI) and EC (Escherichia coli) broth for Staphylococcus aureus and Escherichia coli, respectively. After drying the well, approximately $500 \mu \mathrm{L}$ of the samples resulting from suspensions (PPy I and PPy II) were added to the well and the bacteria were inoculated with the aid of swabs. The resulting plates were incubated at $37^{\circ} \mathrm{C}$ for $24 \mathrm{~h}$ and after registering the inhibition zones, their diameters were measured using the Fiji-ImageJ software. ${ }^{37}$

For MIC determination, the microdilution technique in Mueller-Hinton broth was performed to determine the antibacterial activity. Serial dilutions in 2:2 were performed adding $100 \mu \mathrm{L}$ of the sample solutions in a concentration range of 100 to $12.25 \mathrm{mg} \mathrm{mL}^{-1}$. Subsequently the wells received $20 \mu \mathrm{L}$ of suspensions of microorganisms in each well of the microplates. Gentamicin was used as a positive control in an appropriate concentration for each microorganism. The microplates were incubated in an oven at $37^{\circ} \mathrm{C}$ for $24 \mathrm{~h}$ and the test was performed in triplicate. The resazurin indicator $(100 \mu \mathrm{g} \mathrm{mL}-1)$ was added to each well of the microplates with the presence of the bacteria. The inhibition of microbial growth was evidenced by the lack of growth in the medium, and the MIC was determined to be the lowest concentration of the sample in which no visible growth of bacteria is established in the solution.

\section{Cytotoxicity assay}

The cytotoxicity assay was performed according to adaptations of the ISO 10993:5 $5^{39}$ protocol using the L929 fibroblast cell line. The cells were cultured in Dulbecco's modified eagle medium (DMEM) culture medium (SigmaAldrich, São Paulo, Brazil), supplemented with 10\% FBS (fetal bovine serum), $2 \%$ penicillin/streptomycin solution and kept in an incubator at $37{ }^{\circ} \mathrm{C}$, with $\mathrm{CO}_{2}$ atmosphere by $5 \%$. The polymeric samples were extracted according to modifications of the ISO $10993-12^{40}$ protocol. The standard protocol procedure uses $0.2 \mathrm{~g}$ of the polymer for $1 \mathrm{~mL}$ of culture medium. Both PPy samples showed a fluffy physical structure and were difficult to separate during extraction. Due to this, approximately $50 \mathrm{mg}$ of the samples were used immersed in closed chemically inert containers containing $1000 \mu \mathrm{L}$ of culture medium, using an aseptic technique at $37 \pm 1{ }^{\circ} \mathrm{C}$ under agitation for $24 \mathrm{~h}$. The resulting extracts were assigned to a 96-well plate containing $5 \times 10^{5}$ cells well $^{-1}$. For the negative control, the same number of cells was used without the samples in the plates, while as a positive control, a culture medium containing 10\% dimethyl sulfoxide (DMSO) was used, beyond the empty well containing only the culture medium (blank). Cell viability was obtained in triplicate using the colorimetric method of 3-(4,5-dimethylthiazol-2-yl)2,5-diphenyl tetrazolium bromide (MTT). Absorbance was measured at $570 \mathrm{~nm}$ using the optical density reading in an automated plate reader (ELISA). The results of the cytotoxicity assay are presented as a percentage of viability of the cells grown in the presence of the polymeric extracts compared to the cells grown in a medium without the extracts of the tested materials.

\section{Statistical analysis}

All results from the biological studies were retrieved from three independent experiments and expressed as 
the mean \pm standard deviation $(\mathrm{SD})$, where the error bars in the graphs represent SDs. The differences considered statistically significant were evaluated by ANOVA analysis of variance with the $F$ test in a $95 \%$ confidence interval for $p<0.05$.

\section{Results and Discussion}

\section{Structure}

The powder XRD data for PPy I and PPy II samples are shown in Figure 2. Both diffractograms showed features similar to previous studies reported with PPy. ${ }^{8,41}$ The main difference between the two patterns can be observed in the region between $2 \theta=15-27^{\circ}$ where signals of a higher degree of ordering are present for sample PPy II. This can be due to the use of MO as additive during the preparation.

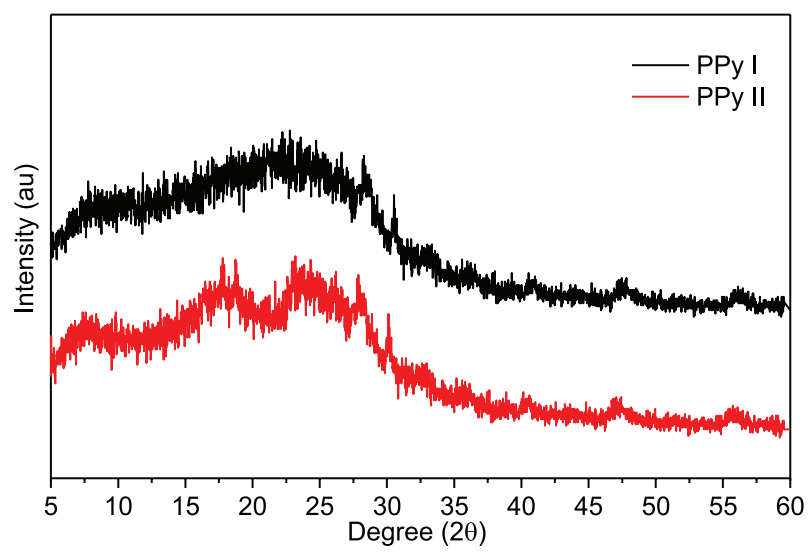

Figure 2. X-ray diffraction pattern of different types of polypyrrole.

For the PPy I sample, XRD pattern is characterized by a broad halo centered at $20^{\circ}, 8,41$ due to the amorphous nature of the conductive polymer. This result is consistent with the formation of non-crystalline PPy, as described from Shrikrushna et al. ${ }^{42}$ da Silva Jr. et al.,${ }^{8}$ and Elnahrawy et al.,${ }^{43}$ On the other hand, PPy II presents two weak and broad peaks between the $15-20^{\circ}$ and $20-27^{\circ 44-47}$ indicative of the presence of regions with partial ordering between chains. Similar features were reported by Zhang and co-workers, ${ }^{44}$ Yang et al., ${ }^{45} \mathrm{Li}$ et al., ${ }^{47}$ in addition to the review by Stejskal and Trchová. ${ }^{15}$

FTIR spectra, Figure 3a, were measured in order to evaluate the functional groups present in PPy samples. ${ }^{48-55}$ Figure $3 \mathrm{~b}$ shows the molecular structures of PPy and MO along with the wavenumber values of relevant chemical bonds.

According to the spectra, the main bands related to the presence of PPy are observed at 1547 and $1459 \mathrm{~cm}^{-1}$ (stretching vibrations of $\mathrm{C}=\mathrm{C}$ and $\mathrm{C}-\mathrm{N}$ bonds of the

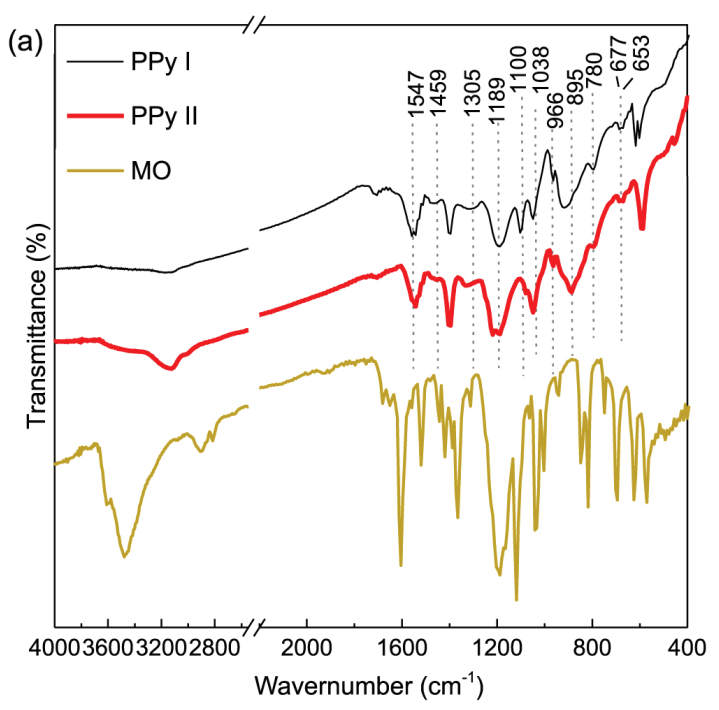

(b)
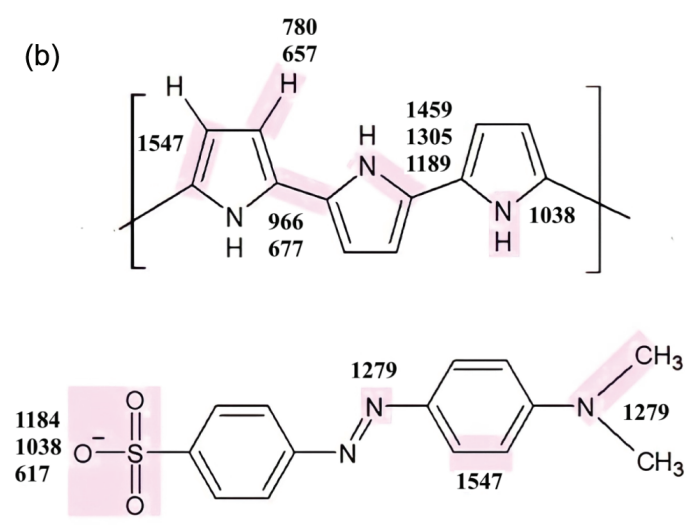

Figure 3. FTIR spectrum for the synthesis of PPy I and PPy II (a) and representation of the wavenumber of the locations of the chemical bonds of PPy and MO, separately (b).

pyrrole ring $),{ }^{8} 1189$ and $1305 \mathrm{~cm}^{-1}(\mathrm{C}-\mathrm{H}$ and $\mathrm{C}-\mathrm{N}$ bending vibrations), ${ }^{8,48} 1100 \mathrm{~cm}^{-1}$ (stretching of the $\mathrm{C}-\mathrm{C}$ bond), ${ }^{8}$ at $1038 \mathrm{~cm}^{-1}$ (deformation vibrations in the plane $\mathrm{C}-\mathrm{H}$ and $\mathrm{N}-\mathrm{H}){ }^{52} \mathrm{Also}$, bands at 966 and $677 \mathrm{~cm}^{-1}$ can be assigned to deformation vibrations of the pyrrolic ring outside the $\mathrm{C}-\mathrm{C}$ plane or to $\mathrm{C}-\mathrm{H}$ balance vibrations, ${ }^{53}$ while bands at 780 and $653 \mathrm{~cm}^{-1}$ evidence vibrations of the Py rings corresponding to out of plane $\mathrm{C}-\mathrm{H}$ bending. ${ }^{54}$ Some differences can be observed between PPy I and PPy II spectra, including the presence of bands assigned to MO for PPy II, as the splitting of the band around $1189 \mathrm{~cm}^{-1}$ due to the existence of a $\mathrm{MO}$ band at $1184 \mathrm{~cm}^{-1}$. Also, an additional band in the $\mathrm{CH} / \mathrm{CN}$ bending region (around $1080 \mathrm{~cm}^{-1}$ ) can be seen for PPy I suggesting that structural differences provided higher degree of vibrational freedom for this sample.

Although the MO spectrum exhibits relatively strong absorption bands, the only spectral manifestation of the dye in the spectrum of PPy II is the above-mentioned band at $1184 \mathrm{~cm}^{-1}$. The absence of most of MO bands in the spectrum of PPy II can be understood considering the diluted character of MO in the sample as well as a possible 

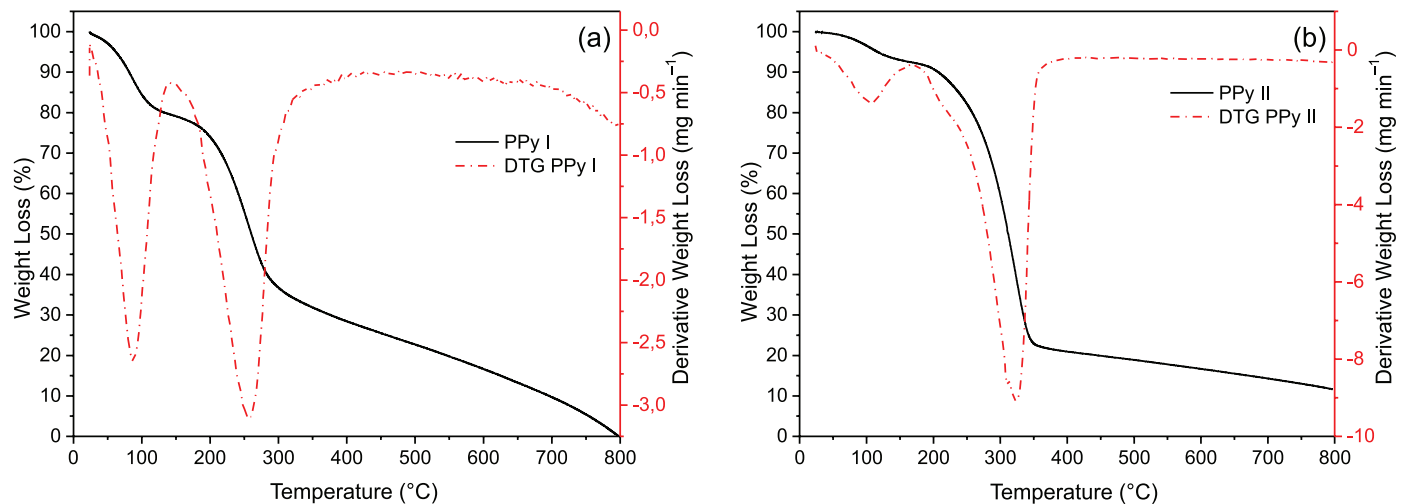

Figure 4. Thermogravimetric analysis (TG) and derivative thermogravimetric (DTG) curves, of resulting synthesis from PPy I (a) and PPy II (b).

predominance of $\mathrm{MO}$ on the surface instead of the bulk of samples. This is in agreement to literature data, ${ }^{54}$ as Sapurina and co-workers ${ }^{54}$ studied the effect of different $\mathrm{MO} /$ pyrrole mole ratios $(0.01,0.05$ and 0.1$)$ as well as the order of addition of the reactants: ( $i$ ) first adding the oxidant to MO solution and then pyrrole; (ii) first adding pyrrole to MO solution and then the oxidant; (iii) the simultaneous addition of pyrrole and oxidant to MO solution. MO bands were detected in FTIR for (ii) and (iii) approaches for all mole ratios, while for (i) only the spectrum of pure PPy is visible till the mole ratio $[\mathrm{MO}] /[$ pyrrole $]=0.1$. Raman spectroscopy, on the other hand, detected MO bands for all concentrations and approaches, as it is a technique that is based on scattering, being thus more sensitive to the surface than FTIR.

The thermal behavior of the samples was evaluated through thermogravimetric analysis (TG) and derivative thermogravimetric (DTG), under nitrogen atmosphere (Figure 4).

The samples present similar decomposition profiles up to the main mass loss, around $25-450{ }^{\circ} \mathrm{C}$. For PPy II, the decomposition is abrupt and well defined, whereas for PPy I the decomposition becomes more gradual with increasing temperature. In the first event (from $25-150^{\circ} \mathrm{C}$ ), the initial mass loss can be attributed to the volatilization of low molecular weight compounds such as water, with mass losses of 12.6 and $7.0 \%$ for PPy I and PPy II, respectively. The curve profiles are similar to studies carried out in the literature ${ }^{55-58}$ and these experimental results could conclude an optimized thermal stability of PPy II in relation to PPy I.

The main difference in thermal behavior of samples from different methodologies can be seen in the second event as those derived from the DTG thermogravimetric curves showed a significant difference in the maximum temperature rate between the two samples. The temperature was higher for PPy II, probably as a result of the greater structural organization, as evidenced by XRD results, increasing the thermal stability of this sample. Finally, the residual mass at the end of curves is also higher for PPy II which can be related with the higher thermal stability too.

\section{Morphology}

As shown in Figure 5, the two samples exhibit distinctive macroscopic appearances. For the PPy I methodology, after mixing the reagents the formation of PPy was evidenced by the color change from colorless to black as well as the formation of a self-standing hydrogel, difficult to remove from the Petri dish.

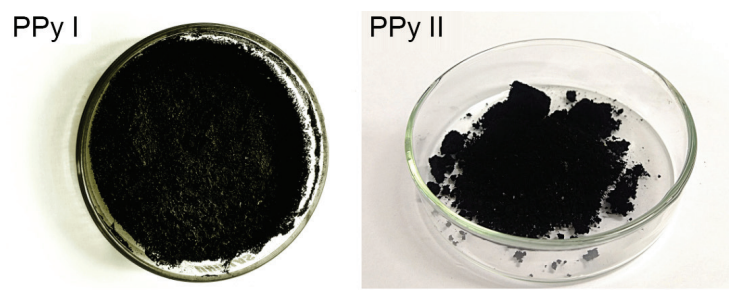

Figure 5. Photographs of PPy I (left) and PPy II (right) samples.

On the other hand, for PPy II, the evidence was a color change from orange to black, with higher yield than for PPy I (approximately $1 \mathrm{~g}$ and $5 \mathrm{~g}$, respectively for PPy I and PPy II). Also, the synthesis and polymerization time is shorter than for PPy I and, as the product is collected by lyophilization, it is fragile and brittle. Microscopic study using SEM highlights the main differences.

The SEM images of samples from both methodologies are shown in Figure 6 and, in general, both samples presented different morphologies due to the different synthesis processing.

PPy I (Figures 6a and 6b) was prepared in the absence of any type of dye, having the typical globular morphology of this polymer, ${ }^{59}$ but with a rough surface and irregular interconnected spheres. The dispersion of diameters is characteristic of an interfacial polymerization. ${ }^{8,60}$ On the other hand, PPy II (Figures 6c and 6d) exhibited a flaky aspect typical of freeze-dried samples. Comparing the 

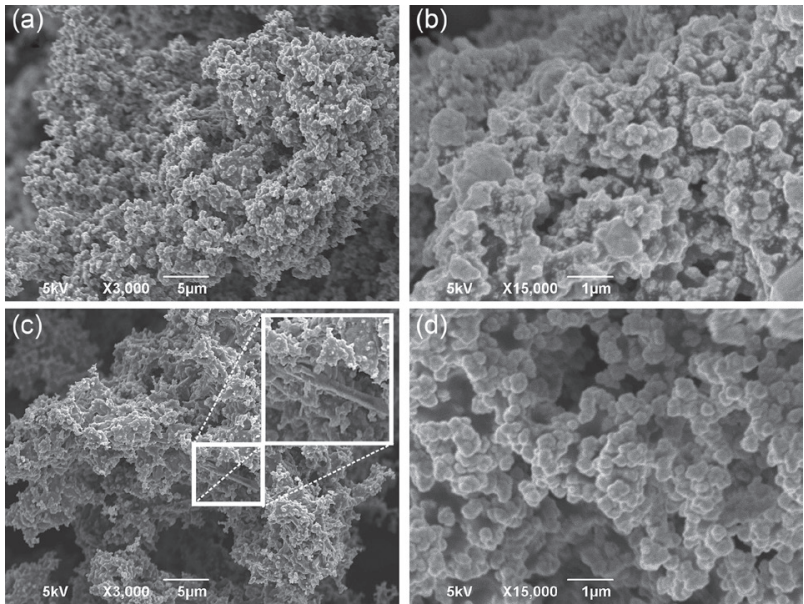

Figure 6. SEM images gives globular PPy morphology of PPy I (a, b) and PPy II (c, d).

two samples (for instance Figure 6b and $6 \mathrm{~d}$ ) the globular morphology of PPy II shows higher regularity due to the presence of MO. ${ }^{15,19,20}$ In both cases, PPy I and PPy II are characterized by a relatively high surface area (BET area of 259.06 and $291.07 \mathrm{~m}^{2} \mathrm{~g}^{-1}$ respectively).

Highlighting Figure 6c (PPy II), the effect of the structure directing agent (MO) is evidenced by the presence of some fibers that can form nanotubes along with the globular aspect. ${ }^{19,20}$ Even using a low MO concentration, the formation of fibers took place in some regions, showing the beginning of the aggregation of globular particles. This process occurs through the use of MO, which can form various types of molecular aggregates, starting with soft micelles, going through rigid or hollow structures, until rearrangements lead to nanotube formation. ${ }^{54}$ In the study from Stejskal and Trchová, ${ }^{15}$ this aggregation depends directly on the type of dye used, and it has been found that MO favors a unidimensional and nanometric morphology. However, some factors can influence this process such as reaction conditions, including temperature, acidity or the introduction of additives. ${ }^{15}$

According to TEM micrographs (Figures 7a-7d) PPy particles from both methodologies showed spherical morphology, in agreement to the globular morphology presented in the SEM micrographs. In general, PPy I show the spherical shapes more clearly than PPy II, which exhibited a higher tendency to form elongated structures. In both cases, particles are coated by a continuous layer of PPy that appear with lower contrast in the images.

For PPy I, the average diameters according to histograms (Figures $7 \mathrm{e}$ and $7 \mathrm{f}$ ) were $25.7 \pm 4.3 \mathrm{~nm}$, demonstrating that the particles were relatively uniform and that the synthesis using PA as single additive stabilizes the material on the nanometric scale. TEM micrographs for PPy II (Figures 7c and 7d) show a predominantly coalesced
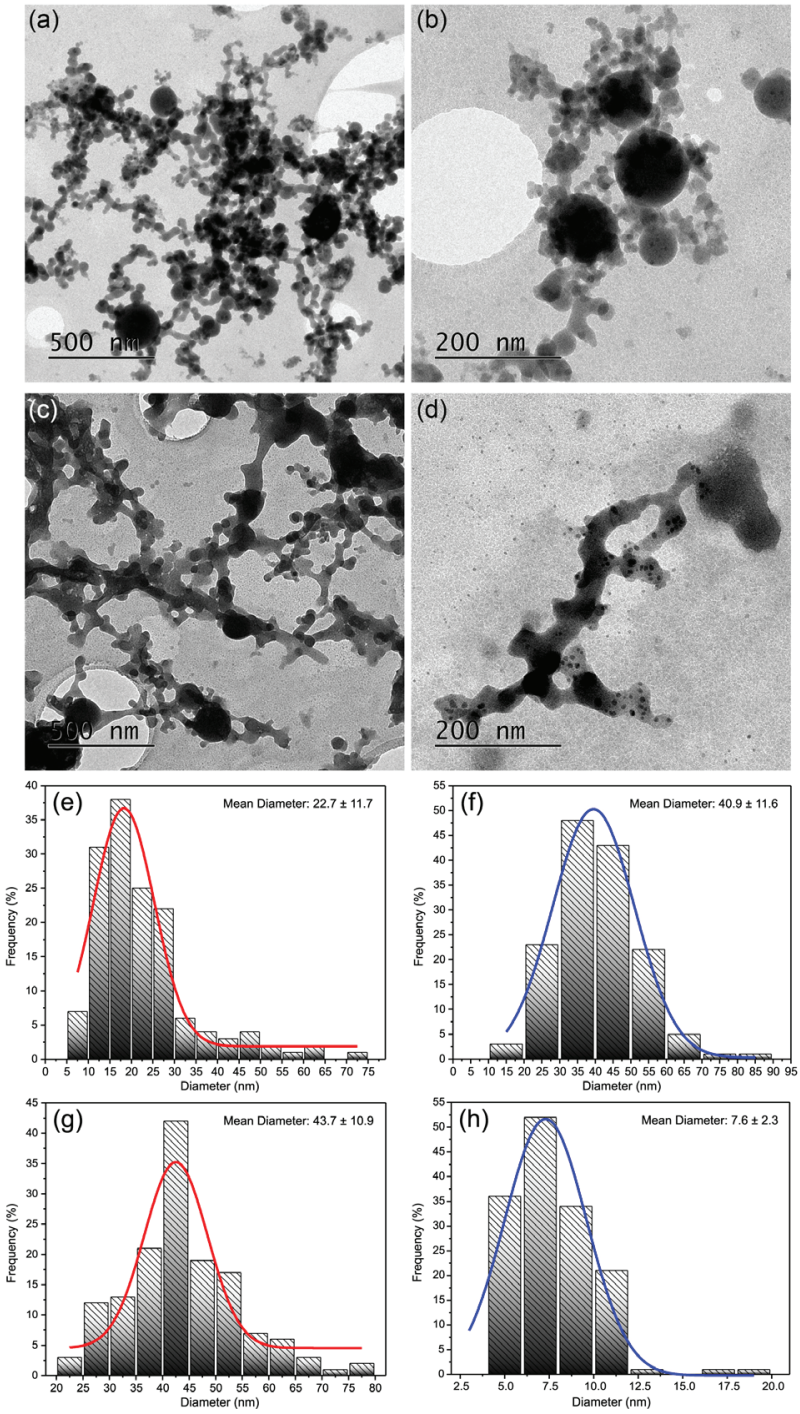

Figure 7. TEM micrograph of PPy I (a, b) and PPy II (c, d) and their respective histograms (e, f to PPy I and g, h to PPy II). The scale bars are 500 and $200 \mathrm{~nm}$ for the two methodologies, respectively. The mean values $\pm \mathrm{SD}$ of the histograms were determined from a Gaussian model.

structure in addition to spheres with an average diameter of $31.8 \pm 0.1 \mathrm{~nm}$. The porous structure of PPy II also looks less dense, with wider free spaces between polymer structures. The presence of elongated structures suggests an effect of the addition of MO into the reaction medium. It is worth to emphasize that, depending on the magnification, SEM images of PPy II also showed the presence of globular particles in addition to some elongated structures. TEM allowed to verify that at high magnifications the elongated structures can be observed in greater detail.

In the literature, ${ }^{19,22,61-63} \mathrm{MO}$ is used as a structural driver as well as an oxidizer, resulting in the formation of PPy globules that decompose into fibers/tubes at a determined concentration of MO, depending on their acid/alkaline behavior. The acidic form of MO favors the spherical form and, for certain concentrations of its basic form, the 
nanotubular form becomes preponderant. The Figure 8 shows the structure of different forms of MO molecules. ${ }^{22}$

(a)

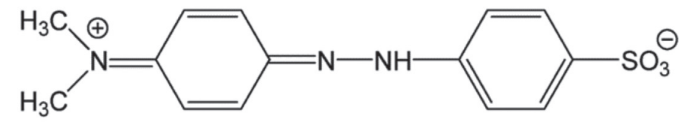

(b)

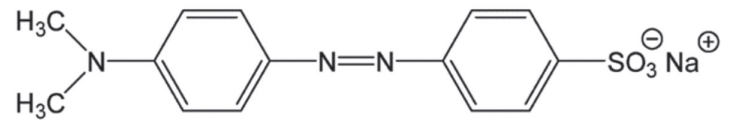

Figure 8. Molecular structure of the MO respectively in acidic (a) and alkaline (b) form.

Sapurina and co-workers ${ }^{54}$ studied the mechanism of formation of PPy nanotubes, proposing that the formation in the presence of MO takes place due to the ability of this dye to form different types of molecular aggregates. Under acidic medium, which can be provided either by acid addition or hydrolysis of some metal salts, MO has limited solubility and forms microneedles able to act as hard templates to nanotubes. However, even in the absence of acidity, MO may have a surfactant-like behavior due to the presence of hydrophilic sulfonic group and large hydrophobic organic chains containing azo groups. This may lead to the formation of supramolecular structures such as micelles playing the role of soft templates to PPy. Finally, nanotube templating from hard and soft route leads to rectangular and circular cross sections, respectively. ${ }^{54}$

\section{Antibacterial activity}

Based on the results from ZI measurement, images of Petri dishes containing the growth inhibition zones for E. coli (E.C.) and S. aureus (S.A.) are shown in Figure 9. The measured values of the ZI and the MIC of the samples are shown in Table 1.

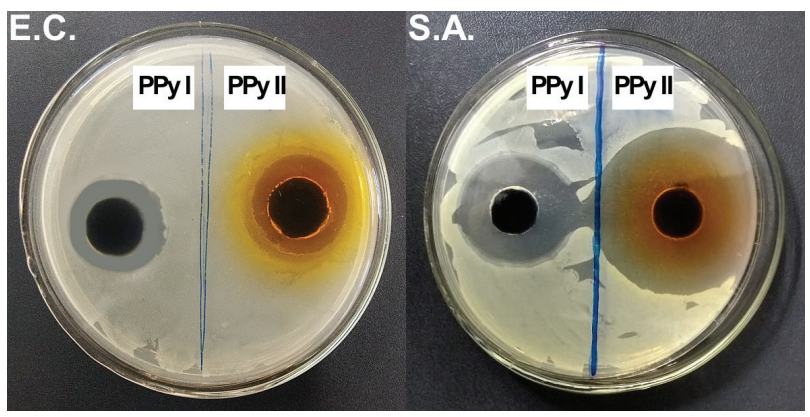

Figure 9. Images of inhibition zones of PPy methodologies against Gram-negative bacteria, E. coli (E.C.) and Gram-positive bacteria, S. aureus (S.A.), after $24 \mathrm{~h}$ of inoculation.

Samples from both methodologies showed great potential as bactericidal agents, although there is no statistical difference in the halo diameter between them
Table 1. ZI diameters for PPy I and PPy II expressed as mean value \pm SD from the triplicate tests and statistical analysis. MIC values were considered, through the use of the resazurin assays, the lowest concentration of each sample capable of inhibiting the growth of $90 \%$ of the strains

\begin{tabular}{lccc}
\hline Bacterial strain & Sample & $\begin{array}{c}\text { Mean ZI diameter } \pm \\
\text { SD } / \mathrm{mm}\end{array}$ & $\begin{array}{c}\text { Mean MIC value / } \\
\left(\mathrm{mg} \mathrm{mL}^{-1}\right)\end{array}$ \\
\hline S. aureus & PPy I & $37.92 \pm 2.08$ & 100 \\
& PPy II & $38.02 \pm 4.93$ & 50 \\
\hline \multirow{2}{*}{. coli } & PPy I & $30.91 \pm 3.78$ & 100 \\
& PPy II & $33.04 \pm 3.46$ & 50 \\
\hline
\end{tabular}

ZI: zone of inhibition; SD: standard deviation; MIC: minimum inhibitory concentration.

and between the bacteria used, considering a confidence level of $p>0.05$ for all relationships. Nevertheless, the sizes of the halos proved that the polymer-microorganism interaction resulted in significant bacterial death, despite the qualitative nature of this analysis.

Based on our observations, it is possible to classify the antibacterial activity of PPy, in terms of inhibition zone, in the following order: Escherichia coli < Staphylococcus aureus. If we consider the efficiency of the bacteriostatic action present in the different PPy syntheses against bacteria, the increasing order is as follows: PPy I E.C. < PPy II E.C. $<$ PPy I S.A. < PPy II S.A.

Comparing the data extracted from Figure 9 and summarized in Table 1, it is evident that the halos were larger for $S$. aureus compared to $E$. coli, irrespective the PPy sample. An explanation to this can be related to the cell wall of Gram-positive bacteria, which has a thick layer of peptidoglycan, while the outer membrane of Gram-negative bacteria has a layer of peptidoglycan and a thin layer of lipopolysaccharide. ${ }^{64-67}$

In this way, we can attribute a more evident bactericidal effect to samples that came in contact with $S$. aureus compared to E. coli. The morphology also has a direct effect on bactericidal activity, since $S$. aureus is characterized by several layers of peptidoglycan and a high degree of porosity, which facilitates the diffusion of PPy particles and the interaction with cells, whereas $E$. coli presents a thin wall that minimizes the diffusion of these particles. ${ }^{64-67}$

The antimicrobial function is the result of some attractive interactions between the cationic groups and the bacterial cell membrane that has a negative charge. These interactions lead to the formation of surfactants causing the disruption of all essential functions of the cell membrane and ceasing the activity of the protein. Another consequence is that bacterial deoxyribonucleic acid (DNA) loses its ability to multiply. Therefore, PPy can be considered a bactericidal agent, since the positive charge in its structure is attracted by the negative charge of the bacterium. Thus, 
the polymeric structure comes into contact with the internal structure of the bacterium, causing its death. ${ }^{64}$

Based on the data in Table 1 for the MIC experiments, regardless of the bacteria used, the MIC was about $100 \mathrm{mg} \mathrm{mL}^{-1}$ for PPy I and about $50 \mathrm{mg} \mathrm{mL}^{-1}$ for PPy II. Comparing the two methodologies for obtaining PPy, the lowest concentration is $50 \mathrm{mg} \mathrm{mL}^{-1}$ assigned to the PPy II sample, which can be explained by the difference between the synthesis, as well as the morphological difference and the addition of additives such as the MO. ${ }^{8,9,67}$

Comparison to literature reports reveals that the materials reported here show similar or higher ZI diameters, in addition to the fact that the bacteriostatic activity for Staphylococcus aureus is significative in comparison to Escherichia coli. For instance, da Silva Jr. et al. ${ }^{8}$ obtained lower ZI diameters for PPy to the same bacteria studied here, even adding silver to potentiate inhibition. In the absence of silver, ZI diameters for S.A. and E.C. respectively were 26.00 and $19.11 \mathrm{~mm}$, whilst in the presence of silver the values were 29.71 and $21.44 \mathrm{~mm}$. Also, they evaluated the antibacterial behavior of PPy as well as the effect of additives on the morphology, observing that the additives and the different experimental procedures changed not only the morphology but also the biological performance.

\section{Cytotoxicity}

In addition to the antibacterial activity investigated in this study, cytotoxicity is another important indicator for assessing the biocompatibility of materials, including polymers. The tests were carried out following ISO 10993-5 standards, with fibroblasts cells, the most commonly used cells to determine the cytotoxicity of polymers. After applying their extracts, the experiments were carried out in triplicate and the resulting average cell viability was calculated in relation to the negative control and blank using equation 1 and the comparison is shown in Figure 10.

Cell viability $(\%)=\frac{\left(\text { Samples }_{570 \mathrm{~nm}}-\text { Blank }_{570 \mathrm{~nm}}\right)}{\left(\text { Negatice control }_{570 \mathrm{~nm}}-\text { Blank }_{570 \mathrm{~nm}}\right)} \times 100$

From the dashed line, all extracts of the two methodologies were found to be non-cytotoxic in comparison to the negative control (without the addition of the samples). In both cases, this indicates that the two methodologies showed biocompatibility $>90 \%$. Statistical analysis revealed that PPy II showed a statistically significant difference relative to both the negative control and PPy I with $p>0.05$.

Both samples synthesized in this study presented satisfactory and significant in vitro tests, however, even

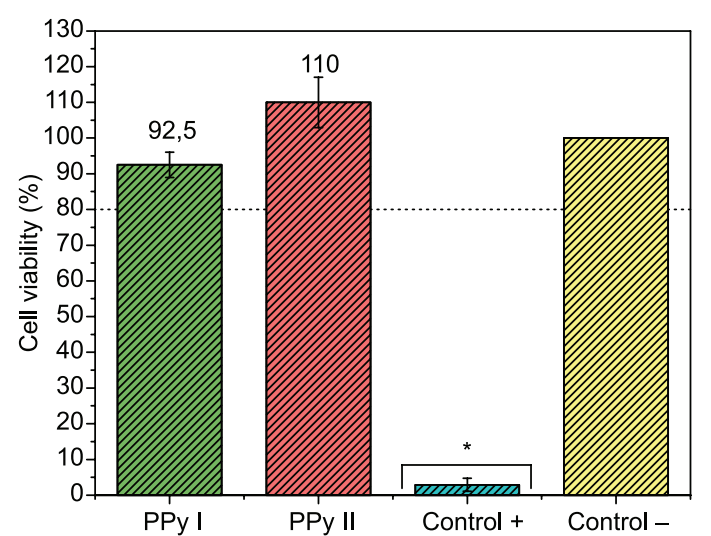

Figure 10. Cytotoxicity of extracts PPy I and PPy II determined on MTT. Asterisks represent $p<0.05$ (by ANOVA analysis of variance with the $F$ test) vs. negative control and dashed lines highlight the limits of feasibility according to ISO 10993-5: viability $>80 \%$ corresponds to no cytotoxicity, $>60-80 \%$ mild cytotoxicity, $>40-60 \%$ moderate cytotoxicity and $<40 \%$ severe cytotoxicity.

obtaining this result, the indications of the methodology from PPy II provide superior results as shown in Figure 10 attributed not only by the difference in synthesis, but also evidence that the morphology alters the results of biological application, similar to the results of microbial application.

Some studies confirm the potential of PPy as a biomaterial, promoting biocompatibility in cytotoxicity tests as in the study by Humpolíček et al. ${ }^{68}$ which compared the biocompatibility of PPy and polyaniline (PANI) with the cytotoxicity and embryotoxicity analysis. Authors obtained results similar to the present study, in addition to the similarity in the cytotoxicity tests using the extract in the MTT assay, as performed here. Another similar work is the study by Zhao et al. ${ }^{29}$ presenting scaffolds of PPy and silk fibroin for repair of neural tissue, with in vitro cytotoxicity tests with L929 cells obtaining significant results such as those presented here.

\section{Conclusions}

The physical-chemical analyzes performed to determine structure (XRD, FTIR and TG) and morphology (BET, SEM and TEM) showed that the two methodologies were effective, confirming the pyrrole polymerization in PPy. In addition, the morphology is directly affected by the experimental procedure and the addition of MO provided satisfactory results for PPy II. The antimicrobial activity resulted in the formation of inhibition halos with significant diameters for the bacteria Escherichia coli and Staphylococcus aureus, indicating that PPy I and PPy II has a potential for application as a biomaterial and can be used as a bactericidal agent with both bacteriostatic (antimicrobial) properties for Grampositive and Gram-negative bacteria. The results of the cytotoxicity assays in vitro allowed to conclude that the two 
methodologies did not result in any cytotoxicity in contact with the fibroblastic cells (L929), but the percentage related to PPy II resulted in the extract that showed the greatest cell viability. However, the modifications made to the syntheses allowed to improve the applicability for incorporation as a biomaterial, providing satisfactory results and demonstrating that the PPy syntheses evidencing their conductive properties and electrical applicability can be effective to be used as an antimicrobial and wound treatment or regeneration agent of muscle tissue.

\section{Acknowledgments}

The authors wish to thank CNPq, CAPES, and FAPITEC network for financial support.

\section{Author Contributions}

Yslaine A. de Almeida was responsible for the conceptualization, data curation, investigation, resources, writing original draft, review and editing; Diego F. Bispo for the investigation and data curation; Monalisa M. Montalvão for the investigation and data curation; Karina O. Mota for the investigation and data curation; Cristiane B. Corrêa for the writing review and editing and visualization; Iara F. Gimenez for the conceptualization, data curation, formal analysis funding acquisition, project administration, resources, writing review and editing.

\section{References}

1. Lima, P. H. C.; Fonseca, D. F.; Braz, C. J. F.; Cunha, C. T. C.; REMAP 2018, 13, 1.

2. Imani, A.; Ghadim, M.; Farzi, G.; J. Nanostruct. Chem. 2014, 4, 101 .

3. Balint, R.; Cassidy, N. J.; Cartmell, S. H.; Acta Biomater. 2014, 10, 2341

4. Pirsa, S.; Shamusi, T.; Mater. Sci. Eng., C 2019, 102, 798.

5. Jain, R.; Jadon, N.; Pawaiya, A.; TrAC, Trends Anal. Chem. 2017, 97, 363.

6. Dey, S.; Kar, A. K.; Mater. Today Proc. 2019, 18, 1072.

7. Dhibar, S.; Roy, A.; Malik, S.; Eur. Polym. J. 2019, 120, 109203.

8. da Silva Jr., F. A. G.; Queiroz, J. C.; Macedo, E. R.; Fernandes, A. W. C.; Freire, N. B.; da Costa, M. M.; de Oliveira, H. P.; Mater. Sci. Eng., C 2016, 62, 317.

9. Upadhyay, J.; Kumar, A.; Gogoi, B.; Buragohain, A. K.; Mater. Sci. Eng., C 2015, 54, 8.

10. Wang, N.; Dai, H.; Wang, D.; Ma, H.; Lin, M.; Mater. Sci. Eng., C 2017, 76, 139.

11. Yang, C.; Mo, H.; Zang, L.; Qiu, J.; Sakai, E.; Wu, X.; Phys. B 2014, 449, 181.
12. Rong, Q.; Han, H.; Feng, F.; Ma, Z.; Sci. Rep. 2015, 5, 11440.

13. Zhu, M.; Li, X.; Ge, L.; Zi, Y.; Qi, M.; Li, Y.; Li, D.; Mu, C.; Mater. Sci. Eng., C 2020, 106, 110185.

14. Ge, X.; He, Y.; Plachy, T.; Kazantseva, N.; Saha, P.; Cheng, Q.; Nanomaterials 2019, 9, 527.

15. Stejskal, J.; Trchová, M.; Chem. Pap. 2018, 72, 1563.

16. Stejskal, J.; Prokeš, J.; Synth. Met. 2020, 264, 116373.

17. Li, Y.; Wang, Y.; Bian, C.; Stejskal, J.; Zheng, Y.; Jing, X.; Dyes Pigm. 2020, 177, 108329.

18. Varga, M.; Kopecká, J.; Moravkova, Z.; Křivka, I.; Trchová, M.; Stejskal, J.; Prokeš, J.; Mor, Z.; Ivo, K.; J. Polym. Sci., Part B: Polym. Phys. 2015, 53, 1147.

19. Kopecká, J.; Mrlík, M.; Olejník, R.; Kopecký, D.; Vrňata, M.; Prokeš, J.; Bober, P.; Morávková, Z.; Trchová, M.; Stejskal, J.; Sensors 2016, 16, 1917.

20. Liang, L.; Chen, G.; Guo, C.-Y.; Mater. Chem. Front. 2017, 1, 380.

21. Sapurina, I.; Li, Y.; Alekseeva, E.; Bober, P.; Trchová, M.; Morávková, Z.; Stejskal, J.; Polymer 2017, 113, 247.

22. Li, Y.; Bober, P.; Trchová, M.; Stejskal, J.; J. Mater. Chem. C 2017, 5, 4236.

23. Stejskal, J.; Chem. Pap. 2020, 74, 1.

24. Capáková, Z.; Radaszkiewicz, K.; Acharya, U.; Truong, T.; Pacherník, J.; Bober, P.; Kašpárková, V.; Stejskal, J.; Pfleger, J.; Lehocký, M.; Humpolíček, P.; Mater. Sci. Eng., C 2020, 113, 110986.

25. Maráková, N.; Humpolíček, P.; Kašpárková, V.; Capáková, Z.; Martinková, L.; Bober, P.; Trchová, M.; Stejskal, J.; Appl. Surf. Sci. 2017, 396, 169.

26. Beyth, N.; Houri-Haddad, Y.; Domb, A.; Khan, W.; Hazan, R.; J. Evidence-Based Complementary Altern. Med. 2015, 2015, 246012

27. Sanchez Ramirez, D. O.; Varesano, A.; Carletto, R. A.; Vineis, C.; Perelshtein, I.; Natan, M.; Perkas, N.; Banin, E.; Gedanken, A.; Mater. Sci. Eng., C 2019, 102, 164.

28. Ezazi, N. Z.; Shahbazi, M. A.; Shatalin, Y. V.; Nadal, E.; Mäkilä, E.; Salonen, J.; Kemell, M.; Correia, A.; Hirvonen, J.; Santos, H. A.; Int. J. Pharm. 2018, 536, 241.

29. Zhao, Y.-H.; Niu, C.-M.; Shi, J.-Q.; Wang, Y.-Y.; Yang, Y.-M.; Wang, H.-B.; Neural Regener. Res. 2018, 13, 1455.

30. Lukášek, J.; Hauzerová, Š.; Havlíčková, K.; Strnadová, K.; Mašek, K.; Stuchlík, M.; Stibor, I.; Jenčová, V.; Řezanka, M.; Polymers 2019, 11, 459.

31. Lalegül-Ülker, Ö.; Elçin, A. E.; Elçin, Y. M. In Advances in Experimental Medicine and Biology; Chun, H.; Park, C.; Kwon, I.; Khang, G., eds.; Springer: Singapore, 2018, p. 135.

32. Álvarez-Paino, M.; Muñoz-Bonilla, A.; Fernández-García, M.; Nanomaterials 2017, 7, 48.

33. Omastová, M.; Bober, P.; Morávková, Z.; Peřinka, N.; Kaplanová, M.; Syrový, T.; Hromádková, J.; Trchová, M.; Stejskal, J.; Electrochim. Acta 2014, 122, 296. 
34. Yuqi, Y.; Asiri, A. M.; Du, D.; Lin, Y.; Analyst 2014, 139, 3055.

35. Pan, L.; Chortos, A.; Yu, G.; Wang, Y.; Isaacson, S.; Allen, R.; Shi, Y.; Dauskardt, R.; Bao, Z.; Nat. Commun. 2014, 5, 3002.

36. Ying, S.; Zheng, W.; Li, B.; She, X.; Huang, H.; Li, L.; Huang, Z.; Huang, Y.; Liu, Z.; Yu, X.; Synth. Met. 2016, 218, 50.

37. Schindelin, J.; Arganda-Carreras, I.; Frise, E.; Kaynig, V.; Longair, M.; Pietzsch, T.; Preibisch, S.; Rueden, C.; Saalfeld, S.; Schmid, B.; Tinevez, J.-Y.; White, D. J.; Hartenstein, V.; Eliceiri, K.; Tomancak, P.; Cardona, A.; Nat. Methods 2012, 9, 676 .

38. Santos, D. C.; Goes, J. M. R.; de Souza, V. C.; Bispo, D. F.; Otubo, L.; Andrade, G. R. S.; Camargo, Z. T.; dos Santos, E. A.; Appl. Surf. Sci. 2020, 505, 144449.

39. ISO 10993-5: Biological Evaluation of Medical Devices, Part 5: Tests for in vitro Cytotoxicity, ISO: Geneva, 2009.

40. ISO 10993-12: Biological Evaluation of Medical Devices, Part 12: Sample Preparation and Reference Materials, ISO: Geneva, 2012.

41. Zhao, J.; Wu, J.; Li, B.; Du, W.; Huang, Q.; Zheng, M.; Xue, H.; Pang, H.; Prog. Nat. Sci.: Mater. Int. 2016, 26, 237.

42. Shrikrushna, S.; Kher, J. A.; Kulkarni, V. M.; J. Nanomed. Nanotechnol. 2015, 6, 1000313.

43. Elnahrawy, A.; Haroun, A.; Hamadneh, I.; Al-Dujaili, A.; Kamel, S.; Carbohydr. Polym. 2017, 168, 182.

44. Li, M.; Yang, L.; Zhang, Y.; RSC Adv. 2015, 5, 1191.

45. Yang, L.; Li, M.; Zhang, Y.; Yi, K.; Ma, J.; Liu, Y.; J. Mater. Sci.: Mater. Electron. 2014, 25, 1047.

46. Wei, D.; Lin, X.; Li, L.; Shang, S.; Yuen, M. C. W.; Yan, G.; Yu, X.; Soft Matter 2013, 9, 2832.

47. Li, M.; Li, W.; Liu, J.; Yao, J.; J. Mater. Sci.: Mater. Electron. 2012, 24, 906.

48. Varga, M.; Kopecký, D.; Kopecká, J.; Křivka, I.; Hanuš, J.; Zhigunov, A.; Trchová, M.; Vrňata, M.; Prokeš, J.; Eur. Polym. J. 2017, 96, 176.

49. Milica, G.; Jugovic, B.; Stevanović, J.; Grgur, B.; Hem. Ind. 2014, 68, 673.

50. Pandey, K.; Saha, P.; Rao, B.; Prep. Biochem. Biotechnol. 2019, 50, 317.

51. Varga, M.; Prokeš, J.; Vr, M.; Trchová, M.; Kopecký, D.; Synth. Met. 2017, 230, 89.
52. Valtera, S.; Prokeš, J.; Kopecká, J.; Vrnata, M.; Trchová, M.; Varga, M.; Stejskal, J.; Kopecký, D.; RSC Adv. 2017, 7, 51495.

53. Liu, J.; Wang, J.; Yu, X.; Shang, S.; Micro Nano Lett. 2015, 10 , 50.

54. Kopecká, J.; Kopecký, D.; Vrňata, M.; Fitl, P.; Stejskal, J.; Trchová, M.; Bober, P.; Moravkova, Z.; Prokeš, J.; Sapurina, I.; RSC Adv. 2014, 4, 1551.

55. Hryniewicz, B. M.; Lima, R. V.; Wolfart, F.; Vidotti, M.; Electrochim. Acta 2019, 293, 447.

56. Yussuf, A.; Al-Saleh, M.; Al-Enezi, S.; Abraham, G.; Int. J. Polym. Sci. 2018, 2018, 4191747.

57. Chen, Y.; Wang, F.; Dong, L.; Li, Z.; Chen, L.; He, X.; Gong, J.; Zhang, J.; Li, Q.; Polymers 2019, 11, 960.

58. Yan, Y.; Li, H.; Zhang, Y.; Kan, J.; Jiang, T.; Pang, H.; Zhu, Z.; Xue, H.; Int. J. Electrochem. Sci. 2017, 12, 9320.

59. Baig, U.; Gondal, M.; Nanosci. Nanotechnol. Lett. 2016, 8, 998.

60. Bo, J.; Luo, X.; Huang, H.; Li, L.; Lai, W.; Yu, X.; J. Power Sources 2018, 407, 105.

61. Gniadek, M.; Malinowska, S.; Rapecki, T.; Stojek, Z.; Donten, M.; Synth. Met. 2014, 187, 193.

62. Liow, K. S.; Sipaut, C. S.; Jafarzadeh, M.; Kai Sing, L.; Sipaut, C. S.; Jafarzadeh, M.; J. Mater. Sci.: Mater. Electron. 2018, 29, 21097.

63. Kaladevi, G.; Meenakshi, S.; Pandian, K.; Wilson, P.; J. Electrochem. Soc. 2017, 164, B620.

64. Silhavy, T. J.; Kahne, D.; Walker, S.; Cold Spring Harbor Perspect. Biol. 2010, 2, a000414.

65. Maitra, A.; Munshi, T.; Healy, J.; Martin, L. T.; Vollmer, W.; Keep, N. H.; Bhakta, S.; FEMS Microbiol. Rev. 2019, 43, 548.

66. Malanovic, N.; Lohner, K.; Biochim. Biophys. Acta, Biomembr. 2016, 1858,936

67. Kumar, R.; Oves, M.; Almeelbi, T.; Al-makishah, N. H.; Barakat, M. A.; J. Colloid Interface Sci. 2017, 490, 488.

68. Humpolíček, P.; Kašpárková, V.; Pacherník, J.; Stejskal, J.; Bober, P.; Capáková, Z.; Radaszkiewicz, K. A.; Junkar, I.; Lehocký, M.; Mater. Sci. Eng., C 2018, 91, 303.

Submitted: November 24, 2020

Published online: February 11, 2021 